\title{
Low-Complexity Switching Network Design for Hybrid Precoding in mmWave MIMO Systems
}

\author{
Francesc Molina and Jordi Borràs \\ Department of Signal Theory and Communications, Technical University of Catalonia (UPC) \\ Email: \{francesc.molina, jordi.borras.pino\}@upc.edu
}

\begin{abstract}
This paper deals with the design of a hybrid precoder for millimeter-wave MIMO systems. For the sake of concreteness, we consider an analog processing stage composed of a switching network with analog combining. The main contribution of this work consists on the proposal and evaluation of an optimization procedure based on a smart relaxation. The optimal hybrid precoder under a transmit power constraint is derived, after which, the analog precoding matrix is binarized. After an intuitive reasoning, we note that multiple solutions exist. Nevertheless, the (very) reduced computational complexity of the proposed optimization scheme makes it feasible for realistic implementations. Numerical results are reported to assess the performance of proposed hybrid precoder design.

Index Terms-Hybrid MIMO, switching network with analog combiner, precoder design, mmWave MIMO.
\end{abstract}

\section{INTRODUCTION}

Spectrum shortage is one of the challenging issues for nextera wireless communications in view of the demanding userrequirements, e.g. high data-rates and low energy consumption, and the expected large amount of wireless interfaces [1], [2]. In this sense, millimeter-wave (mmWave) communications have arisen as a promising candidate for permitting the simultaneous transmission to these massive number of devices [3]. Moreover, to achieve high spectral efficiency communication [4], the use of large-scale multiple-input multiple-output (MIMO) systems [5], [6] is essential. Nevertheless, the implementation of classical baseband (BB) digital combiners in large-scale MIMO poses the challenging problem of energy consumption [7].

Hybrid processing is a promising technology to overcome this problem. The underlying principle of hybrid MIMO technology relies on the use of less radio-frequency (RF) chains than antennas [7-9]. Specifically, the precoder is divided into two stages: the first one consists in a BB digital processor able to support multi-stream transmission; and the second stage is based on purely analog RF processing, which combines the outputs of the RF chains with the transmit antennas. Notice that the decoder works equivalently, first applying the analog processing stage and finally the BB digital processing.

In the recent literature, we may find several works tackling the design of precoders, or decoders, under hybrid MIMO technology [10-16]. Therein, the analog processing stage was

The research leading to this work has been funded by the Spanish Ministry of Science, Innovation and Universities through project WINTER TEC201676409-C2-1-R (AEI/FEDER, UE) and fellowship FPI BES-2017-080071; and by the Catalan Government (AGAUR) under grant 2017 SGR 578 and fellowship FI 2018 supported by the Secretary for Universities and Research of the "Generalitat de Catalunya" and the European Social Fund. initially thought to be implemented using phase shifters. Therefore, the analog combiner was optimized under (un)quantized phase values. A new analog combining approach based on the use of switches, instead of phase shifters, was introduced in [17], In that respect, the analog RF combiner consists in an antenna subset selector carried at less hardware complexity, which yields a reduced power consumption [17], [18]. Additionally, as analyzed in [19], the channel estimation performance of switches-based architectures is equal or better compared to that achieved when using only phase shifters.

It is worth noting that precoding matrices of switches-based hybrid architectures are selection (binary) matrices. Therefore, the optimal analog RF precoder design may be numerically intractable since it involves a combinatorial search due to Boolean constraints. A simple precoder can be implemented under a pure switching architecture, i.e., every RF chain connected to a single antenna. In this case, each column of the analog precoding matrix has a single non-zero element. As addressed in [20], the latter permits exploiting the sparse nature of mmWave channels. However, their inherent performance loss makes it only feasible for small-size antenna arrays.

In this work, we address the last issue. We first consider that hardware complexity and energy consumption are relevant to system design. We adopt a switching network with analog combiner architecture, an extension of pure switching networks where the RF chains are connected to a subset of antennas [19]. For this architecture, the analog precoding matrix constitutes binary columns with an arbitrary number of non-zero elements. As aforementioned, the optimal design may burden computational inefficient, making it unfeasible in practice.

Our contribution is to face the computational complexity considering a hitherto unexplored system optimization based on three stages. We first relax the Boolean feasible set of the analog precoder matrix. We constrain its structure under the definition of an activation function lying in the interval $(0,1)$. Secondly, we address a constrained minimization problem to obtain, from the optimum unconstrained linear precoder, the stationary point equations that optimal hybrid architecture must fulfill, when subject to no power loss. Thirdly, a binarization threshold is applied. This approach differs from cited literature papers in which, for the sake of simplicity, unconstrained optimization is tackled, which incurs into performance penalty. Even though matrix-factorization error increases when the constrained optimization is considered, we show the benefit of incorporating such constraint to increase spectral efficiency. 


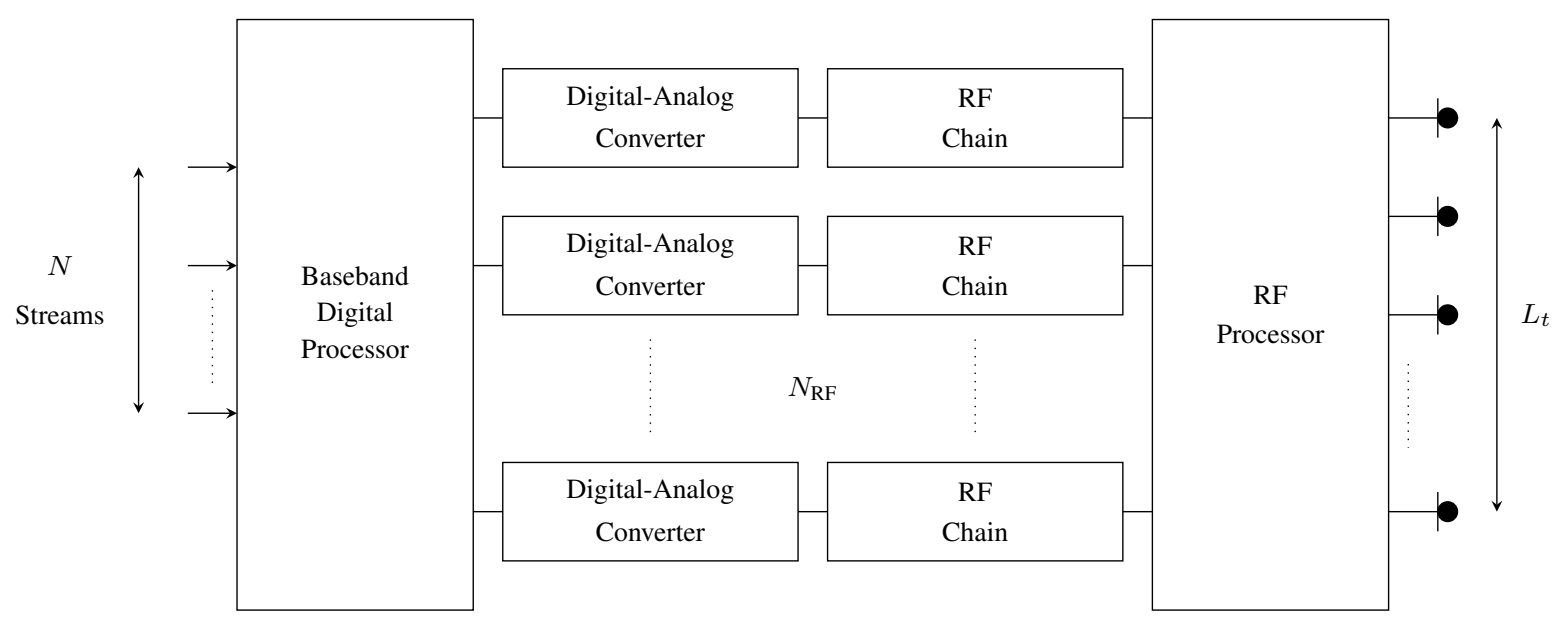

Fig. 1: Hybrid transmitter architecture with $L_{t}$ antennas and $N_{\mathrm{RF}}$ analog chains, with $N \leq N_{\mathrm{RF}} \leq L_{t}$.

The rest of this paper is organized as follows. The system model and the problem statement are presented in Section II. In Section III, we tacle the low-complexity switching network design. Finally, simulation results and conclusions are respectively shown in Sections IV and V.

\section{SySTEM MODEL}

Let us consider the hybrid MIMO transmitter depicted in Fig. 1. We deal with the transmission of $N$ data streams through a point-to-point narrowband ${ }^{1}$ multi-antenna channel $\mathbf{H} \in \mathbb{C}^{L_{r} \times L_{t}}$, with $L_{t}$ and $L_{r}$ being the number of transmit and receive antennas, respectively. For a given precoder matrix $\mathbf{F} \in \mathbb{C}^{L_{t} \times N}$, the received signal $\mathbf{y} \in \mathbb{C}^{L_{r}}$ is given by

$$
\mathbf{y}(n)=\sqrt{\rho} \cdot \mathbf{H F x}(n)+\boldsymbol{v}(n),
$$

with $\mathbf{x}(n) \in \mathbb{C}^{N}$ standing for the transmitted signal at time $n$ with power $\rho$, and $\boldsymbol{v}(n) \sim \mathcal{C N}\left(\mathbf{0} ; \sigma_{v}^{2} \mathbf{I}_{L_{r}}\right)$ is an additive complex white Gaussian noise. Moreover, the mutual information between the transmitter and the receiver can be written as:

$$
I(\mathbf{x} ; \mathbf{y})=\log _{2}\left(\operatorname{det}\left[\mathbf{I}_{L_{r}}+\gamma \cdot \mathbf{H F C}_{\mathbf{x}} \mathbf{F}^{\mathrm{H}} \mathbf{H}^{\mathrm{H}}\right]\right),
$$

with $\mathbf{C}_{\mathbf{x}} \triangleq \mathbb{E}\left\{\mathbf{x}(n) \mathbf{x}^{\mathrm{H}}(n)\right\}=N^{-1} \mathbf{I}_{L_{t}}$, and $\gamma \triangleq \rho \cdot \sigma_{v}^{-2}$. Then, when perfect knowledge of $\mathbf{H}$ is available at the transmitter, the optimal unconstrained $\mathbf{F}_{\text {opt }}$ that maximizes the data rate is

$$
\mathbf{F}_{\text {opt }}=\underset{\mathbf{F}}{\arg \max } I(\mathbf{x} ; \mathbf{y}) \text {. }
$$

The solution is shown to be the first $N$ columns of matrix $\mathbf{V}$ associated to the highest singular values of $\mathbf{H}=\mathbf{U} \boldsymbol{\Sigma} \mathbf{V}^{\mathrm{H}}$. Nevertheless, given the relevance of energy consumption in our design, we consider a hybrid architecture characterized by a BB precoder $\mathbf{F}_{\mathrm{BB}} \in \mathbb{C}^{N_{\mathrm{RF}} \times N}$, and an RF precoder $\mathbf{F}_{\mathrm{RF}} \in$ $\mathbb{C}^{L_{t} \times N_{\mathrm{RF}}}$. Thus, $\mathbf{y}(n)$ is, when $\mathbf{F}$ is substituted by $\mathbf{F}_{\mathrm{RF}} \mathbf{F}_{\mathrm{BB}}$,

$$
\mathbf{y}(n)=\sqrt{\rho} \cdot \mathbf{H F}_{\mathrm{RF}} \mathbf{F}_{\mathrm{BB}} \mathbf{x}(n)+\boldsymbol{v}(n) .
$$

Based on the mutual information (2), the precoder design problem becomes the joint optimization under $\mathbf{F}_{\mathrm{RF}}$ and $\mathbf{F}_{\mathrm{BB}}$ :

$$
\max _{\left\{\mathbf{F}_{\mathrm{RF}} \cdot \mathbf{F}_{\mathrm{BB}}\right\}} \log _{2}\left(\operatorname{det}\left[\mathbf{I}_{L_{r}}+\gamma \cdot \mathbf{H} \mathbf{F}_{\mathrm{RF}} \mathbf{F}_{\mathrm{BB}} \mathbf{F}_{\mathrm{BB}}^{\mathrm{H}} \mathbf{F}_{\mathrm{RF}}^{\mathrm{H}} \mathbf{H}^{\mathrm{H}}\right]\right)
$$

\footnotetext{
${ }^{1}$ The extension to wideband scenarios is straightforward by considering the MIMO-OFDM model presented in [21].
}

Notwithstanding, as shown in [22], the objective function in (5) can be approximated, under certain conditions, by

$$
\min _{\left\{\mathbf{F}_{\mathrm{RF}} \cdot \mathbf{F}_{\mathrm{BB}}\right\}}\left\|\mathbf{F}_{\mathrm{opt}}-\mathbf{F}_{\mathrm{RF}} \mathbf{F}_{\mathrm{BB}}\right\|_{F}^{2},
$$

i.e., minimize the difference (in terms of Frobenius norm) between the optimal unconstrained precoder in (3) and the compound hyrbid precoder, given by the product of $\mathrm{BB}$ and $\mathrm{RF}$ precoding matrices $\mathbf{F}_{\mathrm{RF}} \mathbf{F}_{\mathrm{BB}}$. Intuitively, zero error is achieved as long as the factorization $\mathbf{F}_{\mathrm{opt}}=\mathbf{F}_{\mathrm{RF}} \mathbf{F}_{\mathrm{BB}}$ is permitted, which is, in general, not possible. In this work, we consider that $\mathbf{F}_{\mathrm{RF}}$ has the specific structure to characterize a switching network with an analog combiner [19]. Thus, we address the joint design of $\mathbf{F}_{\mathrm{RF}}$ and $\mathbf{F}_{\mathrm{BB}}$ in agreement with hardware constraints imposed by the adopted hybrid architecture.

\section{Hybrid PRecoder Design}

Given the unconstrained precoder $\mathbf{F}_{\text {opt }}$, we design the precoding matrices: (i) $\mathbf{F}_{\mathrm{RF}} \in \mathcal{B}^{L_{t} \times N_{\mathrm{RF}}}$ under the Boolean alphabet $\mathcal{B}=\{0,1\}$; and (ii) $\mathbf{F}_{\mathrm{BB}} \in \mathbb{C}^{N_{\mathrm{RF}} \times N}$ subject to constraint (8) where $\left\|\mathbf{F}_{\text {opt }}\right\|_{F}^{2}=Q$. Hence, the following pseudoboolean minimization problem is stated:

$$
\begin{array}{rc}
\min _{\left\{\mathbf{F}_{\mathrm{RF}}, \mathbf{F}_{\mathrm{BB}}\right\}} & \left\|\mathbf{F}_{\mathrm{opt}}-\mathbf{F}_{\mathrm{RF}} \mathbf{F}_{\mathrm{BB}}\right\|_{F}^{2} \\
\text { s.t. } & \left\|\mathbf{F}_{\mathrm{RF}} \mathbf{F}_{\mathrm{BB}}\right\|_{F}^{2}=Q \\
\text { s.t. } & {\left[\mathbf{F}_{\mathrm{RF}}\right]_{i, j} \in \mathcal{B}, 1 \leq i \leq L_{t}, 1 \leq j \leq N_{\mathrm{RF}}} \\
\text { s.t. } & {\left[\mathbf{F}_{\mathrm{BB}}\right]_{k, l} \in \mathbb{C}, 1 \leq k \leq N_{\mathrm{RF}}, 1 \leq l \leq N}
\end{array}
$$

It is easy to see that a combinatory problem brought to light w.r.t. $\mathbf{F}_{\mathrm{RF}}$, unfeasible to tackle at large $L_{t}$. For that reason, we may relax $\left[\mathbf{F}_{\mathrm{RF}}\right]_{i, j}$ in (9) to lie in the interval $(0,1)$, instead of $\{0,1\}$, by imposing certain structure over $\mathbf{F}_{\mathrm{RF}}$. To that end, we consider a continuously differentiable increasing function

$$
f: \mathbb{R} \longrightarrow(0,1),
$$

such that $\mathbf{F}_{\mathrm{RF}}$ can be expressed as $\mathbf{F}_{\mathrm{RF}} \triangleq f(\boldsymbol{\Omega})$. Thus, the previous problem (7)-(10) turns out to

$$
\min _{\left\{\boldsymbol{\Omega}, \mathbf{F}_{\mathrm{BB}}\right\}}\left\|\mathbf{F}_{\mathrm{opt}}-f(\boldsymbol{\Omega}) \mathbf{F}_{\mathrm{BB}}\right\|_{F}^{2} \text { s.t. }\left\|f(\boldsymbol{\Omega}) \mathbf{F}_{\mathrm{BB}}\right\|_{F}^{2}=Q
$$


where $[\boldsymbol{\Omega}]_{i, j} \in \mathbb{R}$ and $\left[\mathbf{F}_{\mathrm{BB}}\right]_{k, l} \in \mathbb{C}$. For easy readability, we devote the rest of this Section to highlighting the results we obtained from the optimization we undertake. The explicit mathematical analysis has been moved to the Appendix.

\section{A. Problem Solution}

The solution of our problem is cast in terms of $\mathbf{F}_{\mathrm{RF}}$ and $\mathbf{F}_{\mathrm{BB}}$ that fulfill the following equations:

(i) For a given $\mathbf{F}_{\mathrm{RF}}$, the optimum $\mathbf{F}_{\mathrm{BB}}$ can be obtained as

$$
\mathbf{F}_{\mathrm{BB}}=\varrho^{-1} \cdot \mathbf{F}_{\mathrm{RF}}^{\#} \mathbf{F}_{\mathrm{opt}},
$$

where $\mathbf{F}_{\mathrm{RF}}^{\#}$ is the Moore-Penrose pseudoinverse, and $\varrho$ is a scalar to be determined. Naturally, the structure of the optimum $\mathbf{F}_{\mathrm{BB}}$ coincides with the least squares solution except for the scaling factor

$$
\varrho=Q^{-\frac{1}{2}} \cdot\left\|\mathbf{F}_{\mathrm{RF}} \mathbf{F}_{\mathrm{RF}}^{\#} \mathbf{F}_{\mathrm{opt}}\right\|_{F} .
$$

(ii) For a given $\mathbf{F}_{\mathrm{BB}}$, the optimum $\mathbf{F}_{\mathrm{RF}}=f(\boldsymbol{\Omega})$ is such that

$$
\left.f^{\prime}(\boldsymbol{\Omega}) \odot \mathbb{R e}\left[\left(\mathbf{F}_{\text {opt }}-\varrho \cdot f(\boldsymbol{\Omega}) \mathbf{F}_{\mathrm{BB}}\right) \mathbf{F}_{\mathrm{BB}}^{\mathrm{H}}\right)\right]=\mathbf{0} .
$$

where $\odot$ denotes the Shur-Hadamard product operator, and $\mathbb{R e}[\cdot]$ the real part of the argument.

\section{B. Combining Solutions}

It is clear that the optimum precoder matrices $\mathbf{F}_{\mathrm{RF}}=f(\boldsymbol{\Omega})$ and $\mathbf{F}_{\mathrm{BB}}$ must satisfy, simultaneously, (13) and (15) for the same $\varrho$. Hence, we substitute (13) into (15) to finally get

$$
f^{\prime}(\boldsymbol{\Omega}) \odot\left[\left(\mathbf{I}-\mathbf{F}_{\mathrm{RF}} \mathbf{F}_{\mathrm{RF}}^{\#}\right) \mathbb{R e}\left[\mathbf{F}_{\mathrm{opt}} \mathbf{F}_{\mathrm{opt}}^{\mathrm{H}}\right]\left(\mathbf{F}_{\mathrm{RF}}^{\#}\right)^{T}\right]=\mathbf{0},
$$

which only depends on $\mathbf{F}_{\mathrm{RF}}$ and not on $\varrho, \mathbf{F}_{\mathrm{BB}}$, which highlights that the optimum $\mathbf{F}_{\mathrm{RF}}$ is independent of $Q$. Notice that this fact can be interpreted as follows. According to (13)-(14), $Q$ can be seen as a scaling factor on the BB precoder. Hence, it is independent of the architecture design. From the strict mathematical point of view, the solution does not depend on the specific $f$. In fact, only depends on the optimum $f(\boldsymbol{\Omega})$ lying in $[0,1]$. Nonetheless, from the simulations' perspective, different local optima can be found depending on $f$ given the non-convexity of the optimization problem. In particular, if we choose $f(x)$ to be the Sigmoid $f(x)=\left[1+e^{-a x}\right]^{-1}$ with $a>0$, we have that $f^{\prime}(x)=f(x)(1-f(x))$. Consequently,

$$
f^{\prime}(\boldsymbol{\Omega})=f(\boldsymbol{\Omega}) \odot(1-f(\boldsymbol{\Omega})) .
$$

It is worth noting that $\mathbf{F}_{\mathrm{RF}}$ fulfills (16) in the following cases:

(i) Consider, first of all, that all elements of $f(\boldsymbol{\Omega})$ in (16) achieve the boundary values 0 or 1 . In these particular cases, taking (17) into account, the first element of (16), $f^{\prime}(\boldsymbol{\Omega})$, will result the all-zeros matrix.

(ii) We focus now on the second term in (16), that is $\left(\mathbf{I}-\mathbf{F}_{\mathrm{RF}} \mathbf{F}_{\mathrm{RF}}^{\#}\right) \mathbb{R e}\left[\mathbf{F}_{\mathrm{opt}} \mathbf{F}_{\mathrm{opt}}^{\mathrm{H}}\right]\left(\mathbf{F}_{\mathrm{RF}}^{\#}\right)^{T}$. It is worth pointing out that the square matrix $\mathbf{I}-\mathbf{F}_{\mathrm{RF}} \mathbf{F}_{\mathrm{RF}}^{\#}$ is an orthogonal projection onto the kernel of $\mathbf{F}_{\mathrm{RF}}$. Therefore, whenever all columns of matrix $\mathbb{R e}\left[\mathbf{F}_{\text {opt }} \mathbf{F}_{\text {opt }}^{\mathrm{H}}\right]\left(\mathbf{F}_{\mathrm{RF}}^{\#}\right)^{T}$ can be written as a linear combination of columns in $\mathbf{F}_{\mathrm{RF}}$, viz:

$$
\mathbb{R e}\left[\mathbf{F}_{\text {opt }} \mathbf{F}_{\text {opt }}^{\mathrm{H}}\right]\left(\mathbf{F}_{\mathrm{RF}}^{\#}\right)^{T}=\mathbf{F}_{\mathrm{RF}} \mathbf{A}
$$

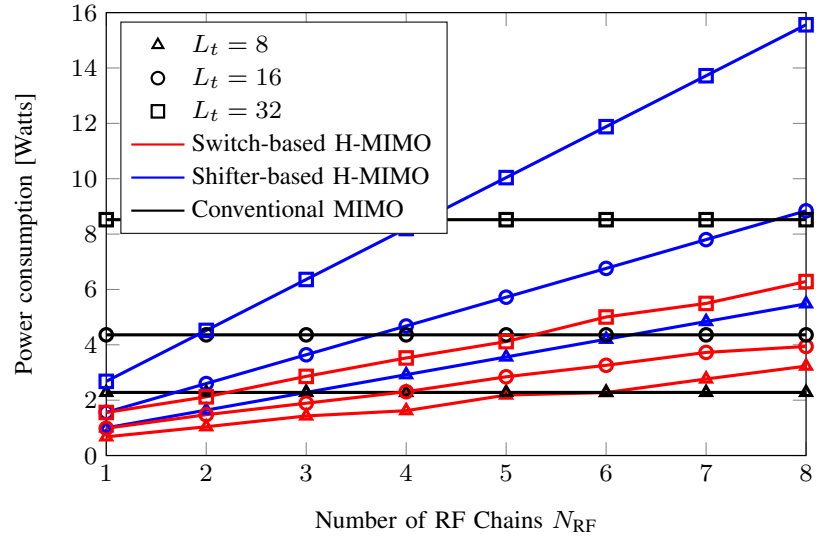

Fig. 2: Power consumption vs. number of RF chains $N_{\mathrm{RF}}$, for different transmitting array's size $L_{t}$. Proposed hybrid precoder (red) is compared with a phase-shifting hybrid precoder (blue) and conventional MIMO (black).

the product $\left(\mathbf{I}-\mathbf{F}_{\mathrm{RF}} \mathbf{F}_{\mathrm{RF}}^{\#}\right) \mathbb{R e}\left[\mathbf{F}_{\mathrm{opt}} \mathbf{F}_{\mathrm{opt}}^{\mathrm{H}}\right]\left(\mathbf{F}_{\mathrm{RF}}^{\#}\right)^{T}$ will be the all-zeros matrix due to orthogonality. Hence, a large number linear combinations $\mathbf{A} \in \mathbb{C}^{N_{\mathrm{RF}} \times N_{\mathrm{RF}}}$ exist, and their performances need to be individually evaluated.

As a conclusion, we observe the following. Albeit the BB precoder can be exactly computed given the RF precoding matrix, we may obtain several local optima for the RF precoder. Mathematically speaking, as also discussed in [14], the solution relies on a saddlepoint. What it is very interesting to highlight is that, as sustained by simulations, local optima present a similar performance in terms of power consumption and spectral efficiency after binarization. Although we have skipped a more sophisticated discussion about the latter due to space limitations, this can be immediately seen from the behavior of non-averaged simulations in Figs. 2 and 3.

\section{Numerical Results}

In this Section, we report a simulation analysis to assess the performance of proposed switching network design. As presented in [22], a very accurate channel model for mmWave MIMO consists in an extension of the Saleh-Valenzuela model. Therefore, we consider the clustered mmWave channel model

$$
\mathbf{H}=\sqrt{\frac{L_{t} L_{r}}{N_{\mathrm{cl}} N_{\text {ray }}}} \sum_{m=1}^{N_{\mathrm{cl}}} \sum_{n=1}^{N_{\mathrm{ray}}} \alpha_{m, n} \mathbf{s}_{\mathrm{R}}\left(\theta_{m, n}\right) \mathbf{s}_{\mathrm{T}}^{\mathrm{H}}\left(\phi_{m, n}\right),
$$

where $\alpha_{m, n}$ stand for the pathloss coefficient, and $\phi_{m, n}, \theta_{m, n}$ for the direction-of-departure (DoD) and direction-of-arrival (DoA) angles, respectively. For the sake of simplicity, and accounting for the poor scattering of the mmWave band, we consider $N_{\mathrm{cl}}=3$ clusters and $N_{\text {ray }}=7$ rays per cluster, where $\phi_{m, n}$ and $\theta_{m, n}$ are uniformly distributed in $[-\pi / 2, \pi / 2]$. For ease of discussion, we adopt, for both the transmitter and the receiver, half-wavelength spaced uniform linear arrays.

The obtained solution (13) and (16) allows decoupling the optimization problem of precoder matrices. We remember local optimum $\mathbf{F}_{\mathrm{RF}}=f(\boldsymbol{\Omega})$ can be found from (16), from which, the optimum $\mathbf{F}_{\mathrm{BB}}$ is computed using (13). As stated before, the Sigmoid parameter $a>0$ may be relevant in simulations for local optima. We set $a=10$ for all cases. 


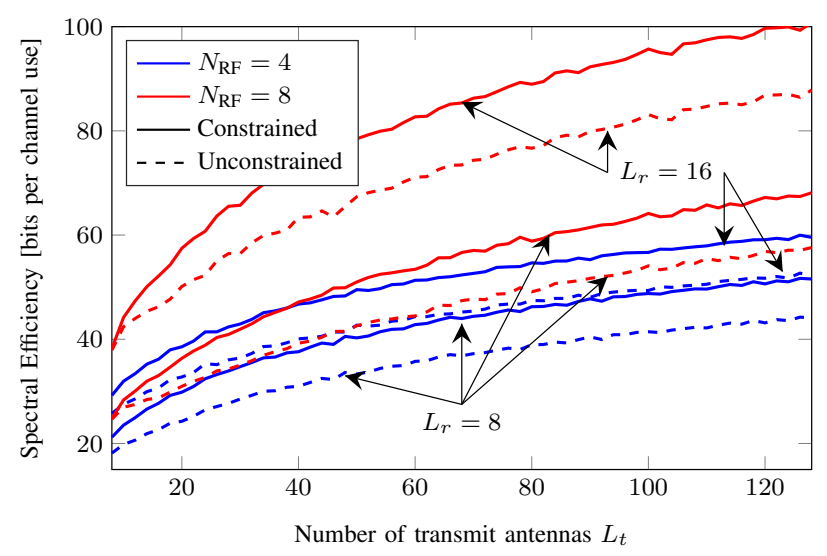

Fig. 3: Spectral efficiency vs. number of transmit antennas $L_{t}$ for different number or RF chains and receiving antennas. Proposed system is compared when using constrained (solid) or unconstrained (dashed) optimization.

\section{A. Power Consumption}

Energy efficiency is one of the main issues of mmWave MIMO. Hence, we analyze the power consumption of proposed switching network-based hybrid precoder. For the sake of simplicity, we consider the power consumption model presented in [19]. Recalling that transmitter is composed of power amplifiers (PA), switches (SW), RF chains (RFC), digital-toanalog converters (DAC), and the $\mathrm{BB}$ combiner, the power consumed by the proposed hybrid architecture is

$$
\begin{aligned}
P_{\text {proposed }} & =\left(L_{t}+N_{\text {switch }}\right) \cdot P_{\mathrm{PA}}+N_{\text {switch }} \cdot P_{\mathrm{SW}} \\
& +N_{\mathrm{RF}} \cdot\left(P_{\mathrm{DAC}}+P_{\mathrm{RFC}}\right)+P_{\mathrm{BB}},
\end{aligned}
$$

where the $N_{\text {switch }}$ is the number of active switches. Notice that it depends on the solution of the stated optimization problem, i.e the number of non-zero elements of the binarized solution.

In Fig. 2, we show the power consumed by the proposed hybrid precoder as a function of the number of RF chains, and for different number of antennas at transmitter $L_{t}$, when the array's size of receiver is fixed to $L_{r}=8$. For the sake of comparison, we compare it with the power consumed by a phase shifting network and conventional MIMO, whose power consumption equation can be found in [19]. We first focus on the switching-based design (red markers). We show that consumed power, although increasing, is always smaller than the power consumed by a shifting network-based hybrid MIMO (for the same number of transmitting antennas). Its great advantage w.r.t. conventional MIMO is appreciated when large transmitting arrays are adopted.

\section{B. Performance Assessment: Error and Spectral Efficiency}

We depict in Fig. 3 the system spectral efficiency as a function of the number of transmit antennas $L_{t}$ for different cases: (i) when using unconstrained and constrained optimization, introduced through variable $Q$ in (12); and (ii) under different RF chains. Similarly as other works, the number of streams is set to $N=N_{\mathrm{RF}}$. The averaged squared error associated to the spectral efficiency values in Fig. 3 is illustrated in Fig. 4. Notice that the number of receiving antennas does not have impact on the squared error. For the sake of simplicity, we have considered a high-SNR regime with uniform power allocation.

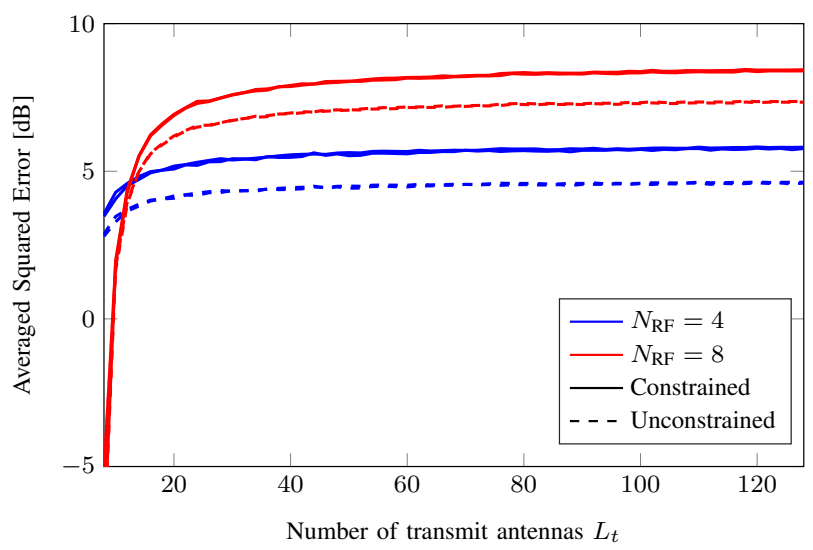

Fig. 4: Squared error ( $\mathrm{dB}$ scale) of the optimization problem, defined as $\left\|\mathbf{F}_{\mathrm{opt}}-\mathbf{F}_{\mathrm{RF}} \mathbf{F}_{\mathrm{BB}}\right\|_{\mathrm{F}}^{2}$, as a function of the transmitting antenna array size for $L_{r}=\{8,16\}$ and different number of RF chains.

Naturally, the solution that minimizes the square error is given by the unconstrained optimization, i.e. setting $\varrho=1$. As shown, constrained optimization leads to significantly increment of system rate in exchange of penalizing the square error. This effect has more impact when $L_{t}, L_{r}$ are low. In particular, at $L_{t}=\{32,64\}$ the system rate is increased by $\{21.6,17.2\} \%$ at $L_{r}=8$ and by $\{17.7,15.7\} \%$ at $L_{r}=16$ when $N_{\mathrm{RF}}=4$. Notice that, since $N_{\mathrm{RF}}=N$, the number of RF chains entails, generally speaking, an increment of system rate and squared error. Yet, we note that, for a small number of transmitting antennas, when $N_{\mathrm{RF}}=N=L_{t}$, the squared error is glaringly reduced. Regarding to the system rate, since the number of streams increases, spectral efficiency is also increased

\section{CONCLUSIONS}

In this work, the design problem of hybrid precoder for millimeter-wave MIMO systems is addressed. Based on the relevance of implementation complexity and energy consumption, we adopt an analog processing stage composed of a switching network with analog combining. To overcome combinatorial optimization, we tackle an optimization problem in which the Boolean alphabet constraints are relaxed to lie in a compact interval. We then derive the stationary point equations of optimal hybrid precoder under a transmit power constraint, after which the optimized analog matrix has to be binarized. We show the relevance of the incorporated power constraint to increase system rate. Numerical results evidence that system rate is further increased when the number of RF chains is low and the number of transmitting and received is moderate.

\section{APPENDIX}

In this Appendix, we tackle the minimization problem (7)(10). For ease of notation, we define $\varepsilon \triangleq\left\|\mathbf{F}_{\mathrm{opt}}-\mathbf{F}_{\mathrm{RF}} \mathbf{F}_{\mathrm{BB}}\right\|_{F}^{2}$, which we develop as follows:

$$
\begin{aligned}
\varepsilon & =\operatorname{Tr}\left[\mathbf{F}_{\mathrm{opt}}^{\mathrm{H}} \mathbf{F}_{\mathrm{opt}}\right]+\operatorname{Tr}\left[\mathbf{F}_{\mathrm{BB}}^{\mathrm{H}} \mathbf{F}_{\mathrm{RF}}^{\mathrm{H}} \mathbf{F}_{\mathrm{RF}} \mathbf{F}_{\mathrm{BB}}\right] \\
& -\operatorname{Tr}\left[\mathbf{F}_{\mathrm{opt}}^{\mathrm{H}} \mathbf{F}_{\mathrm{RF}} \mathbf{F}_{\mathrm{BB}}\right]-\operatorname{Tr}\left[\mathbf{F}_{\mathrm{opt}} \mathbf{F}_{\mathrm{BB}}^{\mathrm{H}} \mathbf{F}_{\mathrm{RF}}^{\mathrm{H}}\right] .
\end{aligned}
$$

The Lagrangian associated with (7)-(8) is

$$
\begin{aligned}
J & =\operatorname{Tr}\left[\mathbf{F}_{\mathrm{opt}}^{\mathrm{H}} \mathbf{F}_{\mathrm{opt}}\right]-\operatorname{Tr}\left[\mathbf{F}_{\mathrm{opt}}^{\mathrm{H}} \mathbf{F}_{\mathrm{RF}} \mathbf{F}_{\mathrm{BB}}\right]-\operatorname{Tr}\left[\mathbf{F}_{\mathrm{opt}} \mathbf{F}_{\mathrm{BB}}^{\mathrm{H}} \mathbf{F}_{\mathrm{RF}}^{\mathrm{H}}\right] \\
& +(1+\lambda) \cdot \operatorname{Tr}\left[\mathbf{F}_{\mathrm{BB}}^{\mathrm{H}} \mathbf{F}_{\mathrm{RF}}^{\mathrm{H}} \mathbf{F}_{\mathrm{RF}} \mathbf{F}_{\mathrm{BB}}\right]-\lambda Q .
\end{aligned}
$$


Hence, the joint optimization under $\mathbf{F}_{\mathrm{BB}}$ and $\boldsymbol{\Omega}$ is addressed in the following sections by deriving, in each of them and for the same $\lambda$, the stationary point equations that each precoder matrix may fulfill when the other remains fixed.

\section{A. Stationary point equation over $\mathbf{F}_{\mathrm{BB}}$}

We take the derivative of $J$ w.r.t. each element in $\mathbf{F}_{\mathrm{BB}}^{\mathrm{H}}$

$$
\frac{\partial}{\partial \mathbf{F}_{\mathrm{BB}}^{\mathrm{H}}}=-\left(\mathbf{F}_{\mathrm{RF}}^{\mathrm{H}} \mathbf{F}_{\mathrm{opt}}\right)^{T}+(1+\lambda)\left(\mathbf{F}_{\mathrm{RF}}^{\mathrm{H}} \mathbf{F}_{\mathrm{RF}} \mathbf{F}_{\mathrm{BB}}\right)^{T}=\mathbf{0} .
$$

Isolating $\mathbf{F}_{\mathrm{BB}}$, we get

$$
\mathbf{F}_{\mathrm{BB}}=\varrho^{-1} \cdot\left(\mathbf{F}_{\mathrm{RF}}^{\mathrm{H}} \mathbf{F}_{\mathrm{RF}}\right)^{-1} \mathbf{F}_{\mathrm{RF}}^{\mathrm{H}} \mathbf{F}_{\mathrm{opt}}=\rho^{-1} \cdot \mathbf{F}_{\mathrm{RF}}^{\#} \mathbf{F}_{\mathrm{opt}},
$$

with $\mathbf{F}_{\mathrm{RF}}^{\#}$ the Moore-Penrose pseudoinverse of $\mathbf{F}_{\mathrm{RF}}$, and $\varrho \triangleq$ $(1+\lambda)=\left\|\mathbf{F}_{\mathrm{RF}} \mathbf{F}_{\mathrm{RF}}^{\#} \mathbf{F}_{\mathrm{opt}}\right\|_{F} Q^{-\frac{1}{2}}$ chosen to satisfy (8).

\section{B. Stationary point equation over $\Omega$}

Recall that we have relaxed the Boolean constraint in (9) by letting $\mathbf{F}_{\mathrm{RF}}=f(\boldsymbol{\Omega})$, where $f(\cdot)$ stands for the a generic increasing function in $[0,1]$. We take the derivative of $J$ (22) w.r.t. $\Omega$ taking as reference the following derivatives in terms of the general matrices $\mathbf{A}$ and $\mathbf{B}$

$$
\begin{aligned}
\frac{\partial}{\partial \boldsymbol{\Omega}} \operatorname{Tr}[\mathbf{A} f(\boldsymbol{\Omega})] & =f^{\prime}(\boldsymbol{\Omega}) \odot \mathbf{A}^{T}, \\
\frac{\partial}{\partial \boldsymbol{\Omega}} \operatorname{Tr}\left[\mathbf{B} f\left(\boldsymbol{\Omega}^{T}\right)\right] & =f^{\prime}(\boldsymbol{\Omega}) \odot \mathbf{B},
\end{aligned}
$$

where $f^{\prime}(\cdot)$ is the derivative of $f(\cdot)$. Notice that (25)-(26) can be easily checked using the chain differentiation rule, with $\odot$ denoting the Shur-Hadamard (element-wise) product operator.

We first differentiate the upper line of (22) in two steps:

1) Firstly, using the invariance of the trace to cyclic permutations, $\operatorname{Tr}\left[\mathbf{F}_{\mathrm{opt}}^{\mathrm{H}} f(\boldsymbol{\Omega}) \mathbf{F}_{\mathrm{BB}}\right]=\operatorname{Tr}\left[\mathbf{F}_{\mathrm{BB}} \mathbf{F}_{\mathrm{opt}}^{\mathrm{H}} f(\boldsymbol{\Omega})\right]$,

$$
\frac{\partial}{\partial \boldsymbol{\Omega}} \operatorname{Tr}\left[\mathbf{F}_{\mathrm{BB}} \mathbf{F}_{\mathrm{opt}}^{\mathrm{H}} f(\boldsymbol{\Omega})\right]=f^{\prime}(\boldsymbol{\Omega}) \odot\left(\mathbf{F}_{\mathrm{opt}}^{*} \mathbf{F}_{\mathrm{BB}}^{\mathrm{T}}\right) .
$$

2) Secondly, $\operatorname{Tr}\left[\mathbf{F}_{\text {opt }} \mathbf{F}_{\mathrm{BB}}^{\mathrm{H}} f\left(\boldsymbol{\Omega}^{T}\right)\right]=\operatorname{Tr}\left[\left(\mathbf{F}_{\text {opt }} \mathbf{F}_{\mathrm{BB}}^{\mathrm{H}}\right)^{T} f(\boldsymbol{\Omega})\right]$,

$$
\frac{\partial}{\partial \boldsymbol{\Omega}} \operatorname{Tr}\left[\left(\mathbf{F}_{\mathrm{opt}} \mathbf{F}_{\mathrm{BB}}^{\mathrm{H}}\right)^{T} f(\boldsymbol{\Omega})\right]=f^{\prime}(\boldsymbol{\Omega}) \odot\left(\mathbf{F}_{\mathrm{opt}} \mathbf{F}_{\mathrm{BB}}^{\mathrm{H}}\right) .
$$

It is worth noting that both are complex conjugate numbers, and thus, the addition of both terms is equivalent to

$$
2 \cdot f^{\prime}(\boldsymbol{\Omega}) \odot \mathbb{R e}\left[\mathbf{F}_{\text {opt }} \mathbf{F}_{\mathrm{BB}}^{\mathrm{H}}\right],
$$

where $\mathbb{R e}[\cdot]$ stands for the real part of the argument.

Finally, the derivative of the lower part of (22) is, using the product differentiation rule

$$
\begin{gathered}
\frac{\partial}{\partial \boldsymbol{\Omega}} \operatorname{Tr}\left[\mathbf{F}_{\mathrm{BB}}^{\mathrm{H}} f\left(\boldsymbol{\Omega}^{T}\right) f(\boldsymbol{\Omega}) \mathbf{F}_{\mathrm{BB}}\right]= \\
f^{\prime}(\boldsymbol{\Omega}) \odot\left(f(\boldsymbol{\Omega}) \mathbf{F}_{\mathrm{BB}}^{*} \mathbf{F}_{\mathrm{BB}}^{\mathrm{T}}\right)- \\
f^{\prime}(\boldsymbol{\Omega}) \odot\left(f(\boldsymbol{\Omega}) \mathbf{F}_{\mathrm{BB}} \mathbf{F}_{\mathrm{BB}}^{\mathrm{H}}\right),
\end{gathered}
$$

which results equivalent to

$$
2 \cdot f^{\prime}\left(\boldsymbol{\Omega}^{T}\right) \odot \mathbb{R e}\left[f(\boldsymbol{\Omega}) \mathbf{F}_{\mathrm{BB}} \mathbf{F}_{\mathrm{BB}}^{\mathrm{H}}\right] .
$$

Therefore, the derivative of $J(22)$ is computed taking into account both (29) and (31). Thus, we get

$$
\left.f^{\prime}(\boldsymbol{\Omega}) \odot \mathbb{R e}\left[\left(\mathbf{F}_{\text {opt }}-\varrho \cdot f(\boldsymbol{\Omega}) \mathbf{F}_{\mathrm{BB}}\right) \mathbf{F}_{\mathrm{BB}}^{\mathrm{H}}\right)\right]=\mathbf{0} .
$$

\section{REFERENCES}

[1] F. Boccardi, R. W. Heath, A. Lozano, T. L. Marzetta, and P. Popovski, "Five disruptive technology directions for 5G," IEEE Commun. Mag., vol. 52, no. 2, pp. 74-80, February 2014.

[2] J. G. Andrews, S. Buzzi, W. Choi, S. V. Hanly, A. Lozano, A. C. K. Soong, and J. C. Zhang, "What will 5G be?" IEEE J. Sel. Areas Commun., vol. 32, no. 6, pp. 1065-1082, June 2014.

[3] S. Sun, T. S. Rappaport, R. W. Heath, A. Nix, and S. Rangan, "MIMO for millimeter-wave wireless communications: beamforming, spatial multiplexing, or both?" IEEE Commun. Mag., vol. 52, no. 12, pp. 110-121, December 2014.

[4] E. Telatar, "Capacity of multi-antenna gaussian channels," European Trans. Telecommun., vol. 10, no. 6, pp. 585-595, 1999.

[5] S. Rangan, T. S. Rappaport, and E. Erkip, "Millimeter-wave cellular wireless networks: Potentials and challenges," Proc. IEEE, vol. 102, no. 3, pp. 366-385, March 2014.

[6] R. W. Heath and A. Lozano, Foundations of MIMO Communication. Cambridge University Press, 2018.

[7] A. Alkhateeb, J. Mo, N. González-Prelcic, and R. W. Heath, "MIMO precoding and combining solutions for millimeter-wave systems," IEEE Commun. Mag., vol. 52, no. 12, pp. 122-131, December 2014.

[8] R. W. Heath, N. González-Prelcic, S. Rangan, W. Roh, and A. M. Sayeed, "An overview of signal processing techniques for millimeter wave MIMO systems," IEEE J. Sel. Topics Signal Process., vol. 10, no. 3, pp. 436-453, April 2016.

[9] X. Gao, L. Dai, S. Han, C. I, and R. W. Heath, "Energy-efficient hybrid analog and digital precoding for mmWave MIMO systems with large antenna arrays," IEEE J. Sel. Areas Commun., vol. 34, no. 4, pp. 9981009, April 2016

[10] A. Alkhateeb, G. Leus, and R. W. Heath, "Limited feedback hybrid precoding for multi-user millimeter wave systems," IEEE Trans. Wireless Commun., vol. 14, no. 11, pp. 6481-6494, November 2015.

[11] F. Sohrabi and W. Yu, "Hybrid digital and analog beamforming design for large-scale antenna arrays," IEEE J. Sel. Topics Signal Process., vol. 10, no. 3, pp. 501-513, April 2016.

[12] D. H. N. Nguyen, L. B. Le, T. Le-Ngoc, and R. W. Heath, "Hybrid MMSE precoding and combining designs for mmWave multiuser systems," IEEE Access, vol. 5, pp. 19 167-19 181, September 2017.

[13] A. F. Molisch, V. V. Ratnam, S. Han, Z. Li, S. L. H. Nguyen, L. Li, and K. Haneda, "Hybrid beamforming for massive MIMO: A survey," IEEE Commun. Mag., vol. 55, no. 9, pp. 134-141, September 2017.

[14] J. Jin, Y. R. Zheng, W. Chen, and C. Xiao, "Hybrid precoding for millimeter wave MIMO systems: A matrix factorization approach," IEEE Trans. Wireless Commun., vol. 17, no. 5, pp. 3327-3339, May 2018.

[15] Y. Huang, J. Zhang, and M. Xiao, "Constant envelope hybrid precoding for directional millimeter-wave communications," IEEE J. Sel. Areas Commun., vol. 36, no. 4, pp. 845-859, April 2018.

[16] Z. Wang, M. Li, Q. Liu, and A. L. Swindlehurst, "Hybrid precoder and combiner design with low-resolution phase shifters in mmwave MIMO systems," IEEE J. Sel. Topics Signal Process., vol. 12, no. 2, pp. 256-269, May 2018.

[17] R. Méndez-Rial, C. Rusu, A. Alkhateeb, N. González-Prelcic, and R. W. Heath, "Channel estimation and hybrid combining for mmWave: Phase shifters or switches?" in 2015 Inf. Theory and App. Work. (ITA), February 2015, pp. 90-97.

[18] E. Zöchmann, S. Schwarz, and M. Rupp, "Comparing antenna selection and hybrid precoding for millimeter wave wireless communications," in 2016 IEEE Sensor Array and Multichannel Signal Process. Work. (SAM), July 2016, pp. $1-5$.

[19] R. Méndez-Rial, C. Rusu, N. González-Prelcic, A. Alkhateeb, and R. W. Heath, "Hybrid MIMO architectures for millimeter wave communications: Phase shifters or switches?" IEEE Access, vol. 4, pp. 247-267, January 2016.

[20] A. Alkhateeb, Y. Nam, J. Zhang, and R. W. Heath, "Massive MIMO combining with switches," IEEE Wireless Commun. Let., vol. 5, no. 3, pp. 232-235, June 2016.

[21] S. Park, A. Alkhateeb, and R. W. Heath, "Dynamic subarrays for hybrid precoding in wideband mmwave MIMO systems," IEEE Trans. Wireless Commun., vol. 16, no. 5, pp. 2907-2920, May 2017.

[22] O. E. Ayach, S. Rajagopal, S. Abu-Surra, Z. Pi, and R. W. Heath, "Spatially sparse precoding in millimeter wave MIMO systems," IEEE Trans. Wireless Commun., vol. 13, no. 3, pp. 1499-1513, March 2014. 